\title{
\begin{tabular}{|l|l|l|l|l|}
\hline M & R & S & Internet Journal of & Nitride Semiconductor Research \\
\hline
\end{tabular}
}

Volume 1, Article 13

\section{Photoluminescence study on GaN homoepitaxial layers grown by molecular beam epitaxy}

\author{
H. Teisseyre, G. Nowak, M. Leszczynski, I. Grzegory, M. Bockowski, S. Krukowski, S. Porowski \\ High Pressure Research Center \\ M. Mayer, A. Pelzmann, Markus Kamp , K. J. Ebeling \\ Abteilung Optoelektronik, Universität Ulm \\ G. Karczewski \\ Institute of Physics, Polish Academy of Sciences
}

This article was received on June 4, 1996 and accepted on September 24, 1996.

\begin{abstract}
GaN epitaxial layers on GaN single crystals were grown using molecular beam epitaxy with an $\mathrm{NH}_{3}$ source. The deposited layers were examined by high resolution $x$-ray diffraction and photoluminescence (PL) spectroscopy. We observed strong and extremely narrow (half-widths of $0.5 \mathrm{meV}$ ) lines related to the bound excitons. In the higher energy range we observed three strong lines. Two of them are commonly attributed to free exciton transitions $\mathrm{A}(3.4785 \mathrm{eV})$ and $\mathrm{B}(3.483 \mathrm{eV})$. Their energetic positions are characteristic of strain-free GaN material.
\end{abstract}

\section{Introduction}

The MOVPE (metalorganic vapor phase epitaxy) technique has dominated research activity in GaN and related materials. This is mainly due to difficulties in supplying atomic nitrogen and achieving high growth rates in the MBE environment, and the improved physical properties of heteroepitaxial layers realized by MOVPE on $\mathrm{Al}_{2} \mathrm{O}_{3}$ and $\mathrm{SiC}$

substrates. The aim of our work was to grow GaN film on GaN single crystals by using the MBE technique, and to compare our samples to homoepitaxial layers grown by MOVPE. To overcome the problem of damage introduced to the epitaxial layer during growth by plasma MBE, we employed the technique of On Surface Cracking (OSC) where uncracked ammonia molecules are injected on to the GaN growth surface. A detailed description of this technique and cracking process which takes place on the sample surface is given in [1].

In this work we report first MBE grown homoepitaxial GaN film. As a substrate we used single GaN crystals grown at about $15 \mathrm{kbar}$ and $1800 \mathrm{~K}$ [2]. The measured $\mathrm{x}$-ray rocking curve full width at half maximum (FWHM) of smallest (about $1 \mathrm{~mm}$ ) substrate crystals used is very close $(\sim 25 \mathrm{arcsec})$ to the theoretical value for a perfect crystals. Larger crystals become slightly mosaic and have small angle boundaries. Photoluminescence (PL) from substrate crystals is influenced by the high free electron concentration $\left(\sim 5 \times 10^{19} \mathrm{~cm}^{-3}\right)$. The ultraviolet peaks are very weak and broadened with a characteristic "shark fin" shaped high energy cut-off corresponding to the position of the Fermi energy (Burstein-Moss effect) [3]. Additionally, a strong yellow luminescence is always observed. The detailed description of physical properties of these crystals is published elsewhere [4]

\section{Experimental results}

For our investigation we used a Riber $32 \mathrm{MBE}$ system adapted for group V gas sources. The system is turbopumped and the attached gas control and handling system is home made. The MBE system is equipped with Knudsen cells used to evaporate $\mathrm{Ga}, \mathrm{Al}$, In and Mg. Ammonia is introduced into the system through a standard high temperature injector (Riber HTI 432). This system was previously used for growing GaN layers on sapphire. The GaN 
single crystals which we used as substrates were mounted on a molybdenum block using indium solder. The growth was performed on the "as grown" substrates without any mechanical or chemical treatment of the surface. Most of the surface was flat and homogeneous.

The structure of the grown layers was examined using high resolution x-ray diffraction. Figure 1 shows the Bragg peaks of the homoepitaxial layer and its substrate. The position of the layer peak is the same as observed in homoepitaxial MOVPE layers [5]. The difference between peaks reflects the much higher concentration of free electrons and point defects in the substrate crystal. The weak and broad shape of the epilayer peak is due to the small $(0.35 \mu \mathrm{m})$ layer thickness.

The PL results were recorded at $4.2 \mathrm{~K}$ with $2 \mathrm{~mW}$ He-Cd laser excitation. The light was dispersed by a Spex $500 \mathrm{M}$ spectrometer and detected by GaAs photomultiplier. The spectrometer was calibrated during each measurement using two ultraviolet lines of an argon laser. The accuracy of energy determination our system is $0.0002 \mathrm{eV}$. Figure 2 shows the PL spectrum of the $0.35 \mu \mathrm{m}$ homoepitaxial layer. We observed that the lines corresponding to the bound excitons are much narrower than the lines corresponding to the free excitons. The lower energy line at $3.4663 \mathrm{eV}$ has a FWHM of $0.5 \mathrm{meV}$. Also, the two next lines at $3.4709 \mathrm{eV}$ and $3.718 \mathrm{eV}$ have comparable FWHM. The relation between intensities of these two lines changes as the excitation point is moved across the epilayer, but in all of our spectra the lines are distinct despite their small $(1 \mathrm{meV})$ energy spacing.

Three next lines at $3.4735 \mathrm{eV}, 3.4785 \mathrm{eV}$ and at $3.483 \mathrm{eV}$ are broader. Their FWHM are roughly $5 \mathrm{meV}$.

We also measured the temperature dependence of our PL spectra. Figure 3 illustrates that with increasing temperature the line at $3.4663 \mathrm{eV}$ becomes broader and weaker. The two next lines at $3.4709 \mathrm{eV}$ and $3.718 \mathrm{eV}$ do not shift, but their intensities also decrease. In the case of the line at $3.4735 \mathrm{eV}$, we observe more significant changes. The intensity decreases with increasing temperature and at $22 \mathrm{~K}$ it nearly vanishes. On the other hand, the intensity of the next line at $3.4785 \mathrm{eV}$ increases with temperature so strongly that it becomes nearly equal to the lines $3.4709 \mathrm{eV}$ and $3.4718 \mathrm{eV}$. Also the intensity of the line at $3.483 \mathrm{eV}$ increases with temperature.

Neither strong yellow luminescence, nor the luminescence near $3.2 \mathrm{eV}$ (donor-acceptor pair transition) was observed.

\section{Discussion and Conclusions}

We have reported preliminary experimental results on the MBE growth of homoepitaxial GaN layers. If we compare our spectra with MOVPE layers we find that we have sharper lines and much stronger free exciton lines.

The narrowest lines can be assigned to the acceptor- $(3.4663 \mathrm{eV})$ and the donor- $(3.4709 \mathrm{eV}$ and $3.4718 \mathrm{eV})$ bound excitons. Their energies are nearly the same as those observed in the MOVPE grown homoepitaxial layers [6]. However, in our case we have sharper lines and can resolve two donor-bound exciton lines. The origin of these two donors is unknown. They can be either native defects or residual impurities.

GaN (wurtzite structure) has a valence band split into three subbands caused by the axial crystal field and the spin-orbit interaction. In optical measurements this valence band splitting gives three free excitons A,B,C [7] [8] [9]. For layers grown on lattice mismatched substrates (sapphire, $\mathrm{SiC}$ ), the exciton lines are broadened and shifted by internal strains. This effect has been studied by e.g. Amano et al. [10] who observed a blue shift of the line corresponding to the exciton bound to a neutral donor. The shift was about $20 \mathrm{meV}$ and this is sufficient to explain differences reported for different samples. In our samples the lines are very narrow and we do not expect any shift related to strain incorporated during the layer growth and subsequent cooling.

The broader lines can be assigned to the free excitons. However, we can not unequivocally identify the $3.4735 \mathrm{eV}$ line. It could be the exciton A, but then the identification of the two other broader lines would be difficult, because they are too close to each other to be related to the valence band splitting. The other explanation (not well confirmed however) is that the line corresponds to the excited state of the donor-bound exciton.

The temperature dependence of the measured spectra strongly supports an assignment of the $3.4785 \mathrm{eV}$ and $3.4830 \mathrm{eV}$ lines as the free excitons, $\mathrm{A}$ and $\mathrm{B}$. The positions of these lines are similar to those previously reported [6] for homoepitaxial MOCVD samples.

In summary, we report the successful MBE growth of a GaN homoepitaxial layer. The very high quality of the layers is confirmed by the $0.5 \mathrm{meV}$ FWHM of the bound exciton lines which allowed us to resolve lines related to excitons bound to different shallow donors. 


\section{References}

[1] M. Kamp, M. Mayer, A. Pelzmann, A. Thies, H. Y. Chung, H. Sternschulte, O. Marti, K. J. Ebeling, Mater. Res. Soc. Symp. Proc. 395, 135-139 (1996).

[2] S. Porowski, I. Grzegory, J. Jun, in High Pressure Chemical Synthesis, Edited by: J. Jurczak, B. Baranowski, (Elsevier, Amsterdam, 1989) 21.

[3] H. Teisseyre, P. Perlin, T. Suski, I. Grzegory, S. Porowski, J. Jun , A. Pietraszko, T. D. Moustakas, J. Appl. Phys. 76, 2429-2434 (1994).

[4] M. Leszczynski, T. Suski, H. Teisseyre, P. Perlin, I. Grzegory, J. Jun, S. Porowski, T. D. Moustakas, J. Appl. Phys. 76, 4909-4911 (1994).

[5] M. Leszczynski, H. Teisseyre, T. Suski, I. Grzegory, M. Bockowski, J. Jun, S. Porowski, K. Pakula, J. M. Baranowski, C. T. Foxon, T. S. Cheng, Appl. Phys. Lett. 69, 73-75 (1996).

[6] K Pakula, A Wysmolek, KP Korona, JM Baranowski, R Stepniewski, I Grzegory, M Bockowski, J Jun, S Krukowski, M Wroblewski, S Porowski, Sol. St. Comm. 97, 919-922 (1996).

[7] R. Dingle, D. D. Sell, S. E. Stokowski, M. llegems, Phys. Rev. B 4, 1211 (1971).

[8] B. Monemar, Phys. Rev. B 10, 676 (1974).

[9] B Gil, O Briot, RL Aulombard, Phys. Rev. B 52, R17028-17031 (1995).

[10] H. Amano, K. Hiramatsu, I. Akasaki, Jpn. J. Appl. Phys. 27, L1384 (1988).

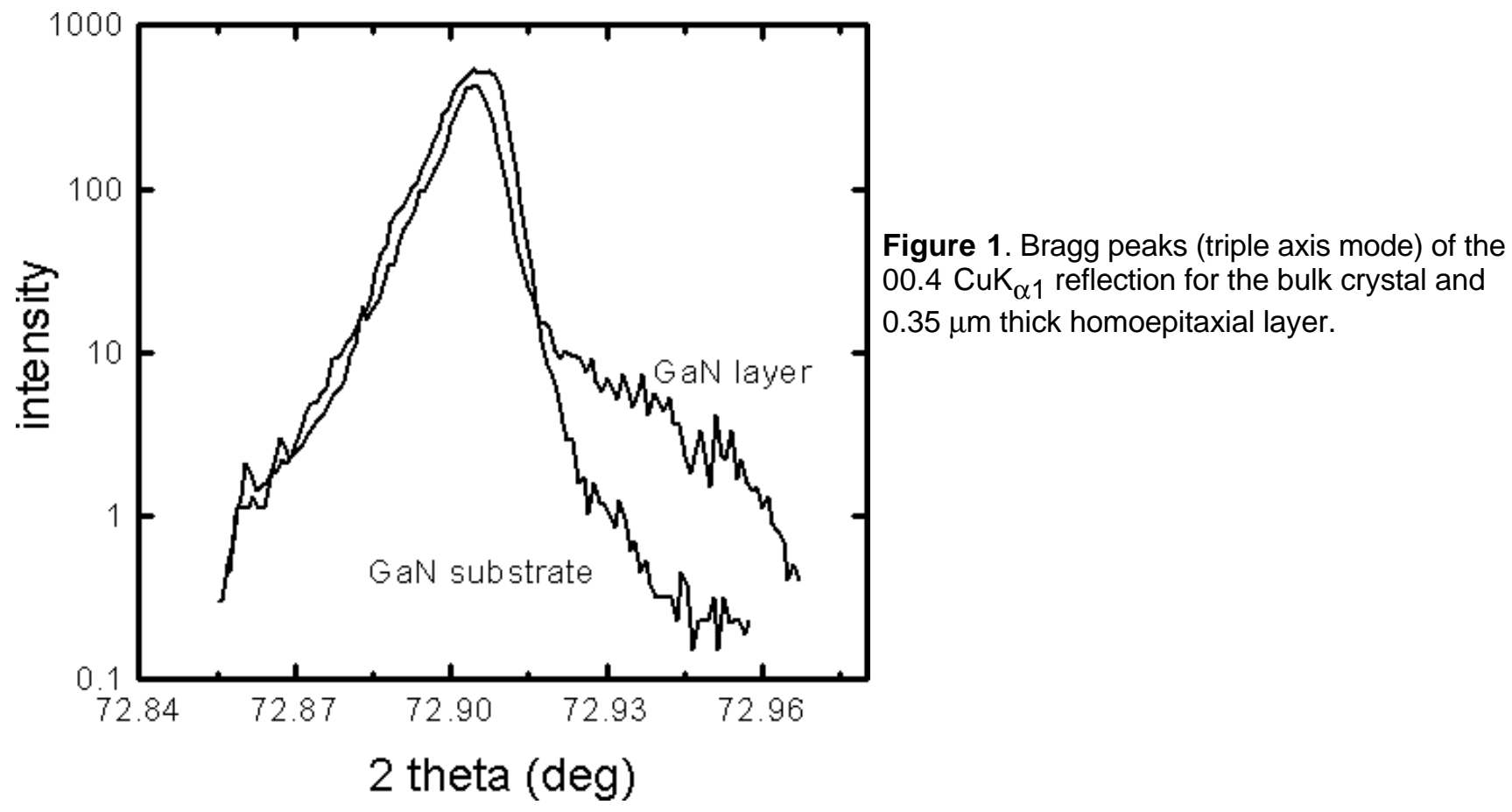




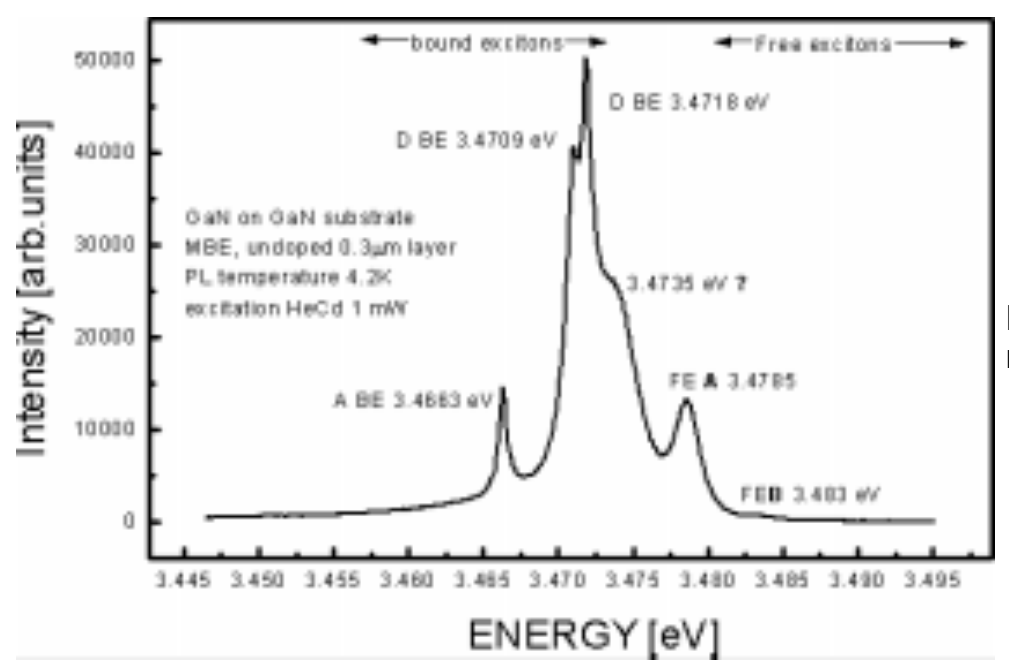

Figure 2. The exciton region of the PL spectrum measured at $4.2 \mathrm{~K}$

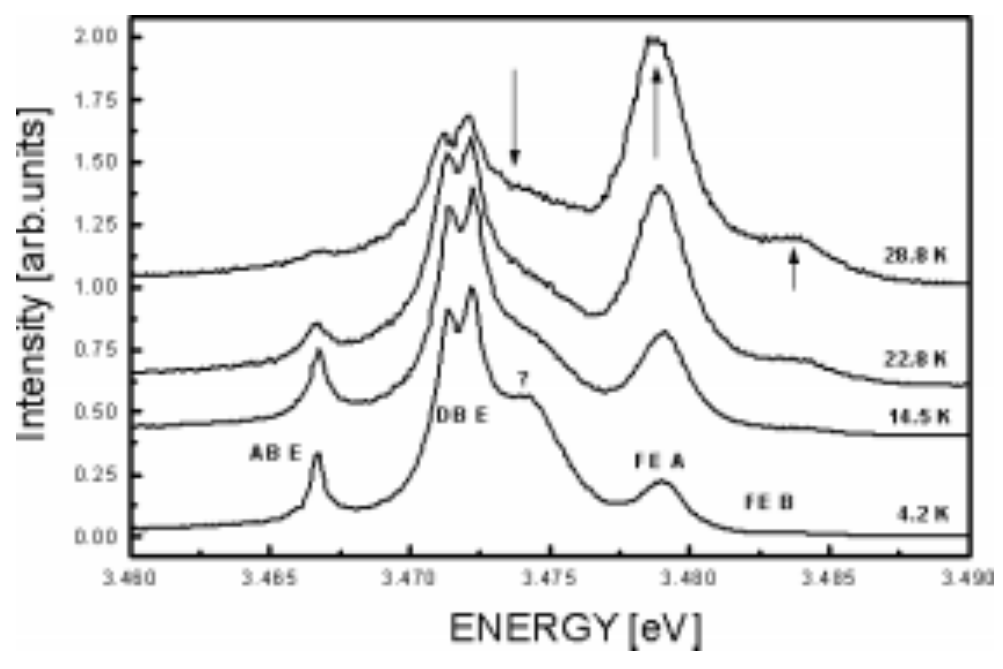

Figure 3. Temperature dependence of the exciton region of the PL spectrum.

(C) 1996-1997 The Materials Research Society

\begin{tabular}{|l|l|l|l|l|}
\hline M & $\mathrm{R}$ & $\mathrm{S}$ & Internet Journal of & Nitride Semiconductor Research \\
\hline
\end{tabular}

